\title{
Regarding invited papers on dynamical systems
}

\author{
Hiroshi Kokubu' ${ }^{1}$. Hisashi Okamoto ${ }^{2}$
}

Published online: 26 June 2015

(C) The JJIAM Publishing Committee and Springer Japan 2015

In 2013, the Research Institute for Mathematical Sciences, Kyoto University, hosted the thematic project "Dynamical Systems: New Directions in Theory and Applications". After its very successful conclusion, Hiroshi Kokubu, the chief organizer, called for original and survey papers representing the outcome of the project.

Four papers were submitted to the present journal, for which $\mathrm{H}$. Kokubu is one of the associate editors. Those four are: "A topological measurement of protein compressibility", by Marcio Gameiro et al., which was published in the last issue; "Topological data analysis and cosheaves", by Justin Michael Curry (present issue); "Common formation mechanism of basin of attraction for bipedal walking models by saddle hyperbolicity and hybrid dynamics", by Ippei Obayashi et al. (present issue); "Smooth parametrizations in dynamics, analysis, diophantine, and computational geometry", by Yosef Yomdin (present issue).

Hisashi Okamoto, the editor for Area 1, is convinced that these four papers represent cutting-edge tools and frontline applications of the theory of dynamical systems, and expresses his deepest gratitude to their authors for spending hours for submission, revision, and publication.

\footnotetext{
¿ Hisashi Okamoto okamoto@kurims.kyoto-u.ac.jp

Hiroshi Kokubu kokubu@math.kyoto-u.ac.jp

1 Department of Mathematics, Kyoto University, Kyoto, Japan

2 Research Institute for Mathematical Sciences, Kyoto University, Kyoto, Japan
} 